\title{
An Expeditious Oxidative Aromatization of Hantzsch 1,4-Dihydropyridines to Pyridines Using Cetyltrimethylammonium Peroxodisulfate: A Phase Transferring Oxidant
}

\author{
Parvin Kumar* and Ashwani Kumar ${ }^{\dagger}$ \\ Chemistry Department, Guru Nanak Khalsa College, Yamuna Nagar, Haryana -135001, India \\ *E-mail:drpkawasthignkc@rediffmail.com \\ ${ }^{\dagger}$ Drug discovery and research laboratory, Department of Pharmaceutical Sciences, \\ Guru Jambheshwar University of Science \& Technology, Hissar, Haryana -125001, India \\ Received May 22, 2010, Accepted June 26, 2010
}

\begin{abstract}
A new approach to the use of potassium peroxodisulphate as an oxidizing reagent is proposed and applied to the case of oxidative aromatization of 1, 4-dihydropyridines with cetyltrimethylammonium peroxodisulfate, a phase transfer oxidant. We demonstrate how it is possible to increase the reactivity of potassium peroxodisulphate in the presence of phase transfer catalyst. Dealkylation in case of $4-n$-alkyl $/ n$-alkenyl was not obtained.
\end{abstract}

Key Words: Oxidative aromatization, Cetyltrimethylammonium peroxodisulfate (CTAPDS), Phase transfer catalyst, 1,4-DHP, Cetyltrimethyl ammonium bromide

\section{Introduction}

The pyridine nucleus is of substantial significance as this ring is the key component in a variety of bioactive compounds, both naturally occurring and synthetic. ${ }^{1}$ Thus, the synthesis of highly substituted pyridines has attracted much attention, and a number of procedures have been developed. ${ }^{2}$ Among these, one of the very convenient approaches which attracted our attention is the oxidative aromatization of 1,4-DHP. The 1,4-dihydropyridine (1,4-DHP) L-type voltage sensitive calcium ion channel represents an important drug target that possesses specific binding sites for both antagonist and agonist ligands that modulate the closed or open conformational state of the channel. ${ }^{3}$ These compounds generally undergo oxidative metabolism in the liver by the action of cytochrome p-450 to form the corresponding pyridine derivatives. ${ }^{4}$ These ubiquitous features always encourage synthetic chemist to explore improved protocols for the synthesis as well as the oxidation of 1,4-DHPs. A variety of reagents has been utilized for this oxidative conversion: S-nitrosoglutathion, ${ }^{5} \mathrm{~N}_{2} \mathrm{O}_{4}$ complex of 18-crown-6, ${ }^{6}$ photochemical oxidation, ${ }^{7} \mathrm{Zr}\left(\mathrm{NO}_{3}\right)_{4},{ }^{8}$ tetrakis-(pyridine)cobalt(II) dichromate, ${ }^{9}$ nicotinum dichromate, ${ }^{10}$ cytochrome P-450, ${ }^{11}$ electrochemical catalysis, ${ }^{12}$ urea nitrate, ${ }^{13}$ peroxydisulfate$\mathrm{Co}(\mathrm{II}),{ }^{13} \mathrm{Mn}(\mathrm{OAc})_{3},{ }^{14}$ Vanadium salts, ${ }^{15}$ tetra- $n$-butylammonium periodate, ${ }^{16} \mathrm{AlCl}_{3},{ }^{17}$ benzyltriphenylphosphonium peroxymonosulfate, ${ }^{18} \mathrm{Mn}$ (TPP)Cl-PSI/NaIO ${ }_{4},{ }^{19}$ urea- $\mathrm{H}_{2} \mathrm{O}_{2},{ }^{20} \mathrm{Fe}$ $\left(\mathrm{ClO}_{4}\right)_{3} / \mathrm{AcOH},{ }^{21} \mathrm{H}_{6} \mathrm{PMo}_{9} \mathrm{~V}_{3} \mathrm{O}_{40},{ }^{22}$ pyridinium chlorochromate, ${ }^{23}$ $\mathrm{K}_{2} \mathrm{FeO}_{4},{ }^{24}$ iodine, ${ }^{25}$ silica modified sulfuric acid/ $\mathrm{NaNO}_{2}$, ${ }^{26}$ $\mathrm{KBrO}_{3} / \mathrm{SnCl}_{4} \cdot 5 \mathrm{H}_{2} \mathrm{O},{ }^{27}$ silica Chromate, ${ }^{28} \mathrm{MnO}_{2},{ }^{29}$ hypervalent iodine. $^{30-35}$ In spite of an overabundance of methods for this conversion, limitations like extended reaction times, poor yields and use of strong or toxic oxidant has led to the investigation of many alternative procedures.

Therefore, it is of practical importance and need of the hour to develop and introduce a convenient, milder and efficient method for the oxidation of 1,4-dihydropyridines to the corresponding pyridines. The peroxodisulfate ion is one of the strongest oxidizing agents known in aqueous solution. The standard oxidation-reduction potential for the reaction is estimated to be $-2.01 \mathrm{~V}$. The oxidative application of peroxodisulfate ion in organic synthesis has been widely investigated. ${ }^{36}$

The main disadvantage associated with the use of potassium peroxodisulphate is the difficulty encountered to solubilize the peroxodisulfate ion in organic solvents. The phase transfer oxidant, tetrabutylammonium peroxodisulfate ${ }^{36(\mathrm{j})}$ and cetyltrimethylammonium peroxodisulfate (CTAPDS), can be used for oxidation of various organic substrates and as free radical generator in organic solvents.

Cetyltrimethylammonium peroxodisulphate, ${ }^{37}$ CTAPDS, a mild, efficient, stable, and cheap reagent, is a white powder quite soluble in most organic solvents such as methanol, ethanol, dichloromethane, chloroform, ethyl acetate, dioxane, benzene, acetone and acetonitrile. This reagent is easily prepared by addition of an aqueous solution of cetyltrimethylammonium bromide to a solution of potassium peroxodisulfate in water.

\section{Result and Discussion}

In continuation to our work on aromatization of 1,4-DHP, ${ }^{34,35}$ we report herein oxidation of 1,4-DHP to corresponding pyridine derivative using cetyltrimethylammonium peroxodisulfate, a phase transfer oxidant (Scheme 1). Results are summarized in Table 1.

In preliminary experiments to study the oxidizing efficiency of reagent CATPDS and substituents effect, we selected the reactions of 4-methoxyphenyl, 4-phenyl and 4-nitrophenyl substituted 1,4-DHP as model reactions with reagent CATPDS in various solvents such as hexane, $\mathrm{CH}_{2} \mathrm{Cl}_{2}, \mathrm{CHCl}_{3}, \mathrm{CH}_{3} \mathrm{CN}$ and $\mathrm{THF}$ at room temperature and refluxing conditions. The oxidative aromatization of 1,4-DHP was not successful at room 
<smiles>[R]C1C(COCC)=C(C)NC(C)=C1OCC</smiles>

1 cetyltrimethylammonium peroxodisulfate (1.2 equivalent)<smiles>[R]c1c(COCC)c(C)nc(C)c1C(=O)OCC</smiles>

2<smiles>CCOC(=O)c1cc(C)nc(C)c1C(=O)OCC</smiles>

3

1. $\mathrm{R}=$ (a) $\mathrm{C}_{6} \mathrm{H}_{5}$; (b) 4- $\mathrm{NO}_{2} \mathrm{C}_{6} \mathrm{H}_{4}$; (c) 3- $\mathrm{NO}_{2} \mathrm{C}_{6} \mathrm{H}_{4}$; (d) $2-\mathrm{NO}_{2} \mathrm{C}_{6} \mathrm{H}_{4}$; (e) 4- $\mathrm{MeOC}_{6} \mathrm{H}_{4}$; (f) $3,4\left(\mathrm{OCH}_{3}\right)_{2} \mathrm{C}_{6} \mathrm{H}_{3}$; (g) 4- $\mathrm{CH}_{3} \mathrm{C}_{6} \mathrm{H}_{4}$; (h) 4-ClC $\mathrm{H}_{4}$ (i) 2,4- $\mathrm{Cl}_{2} \mathrm{C}_{6} \mathrm{H}_{3}$; (j) 3- $\mathrm{BrC}_{6} \mathrm{H}_{4}$; (k) 3-PhCOO- $\mathrm{C}_{6} \mathrm{H}_{4}$; (l) $\mathrm{C}_{6} \mathrm{H}_{5} \mathrm{CH}=\mathrm{CH}$; (m) $\mathrm{CH}_{3} \mathrm{CH}=\mathrm{CH}$; (n) 2-Furyl; (o) 3-Pyridyl; (p) 2-Thienyl; (q) $\mathrm{CH}_{3}$; (r) $\mathrm{C}_{2} \mathrm{H}_{5}$; (s) $\mathrm{CH}_{3} \mathrm{CH}_{2} \mathrm{CH}_{2}$; (t) $\mathrm{CH}_{3} \mathrm{CH}_{2} \mathrm{CH}_{2} \mathrm{CH}_{2}$; (u) $\mathrm{C}_{6} \mathrm{H}_{13}$; (v) $\left(\mathrm{CH}_{3}\right)_{2} \mathrm{CH}$; (w) $\mathrm{C}_{6} \mathrm{H}_{5} \mathrm{CH}_{2}$; (x) $\mathrm{C}_{6} \mathrm{H}_{11}$ (cyclohexyl); (y) $\mathrm{C}_{6} \mathrm{H}_{9}$ (cyclohex-3-enyl); (z) $\mathrm{C}_{6} \mathrm{H}_{5}-\mathrm{CH}_{-} \mathrm{CH}_{3} ;$ (aa) $\mathrm{H}$.

2. $\mathrm{R}=\left(\right.$ a) $\mathrm{C}_{6} \mathrm{H}_{5}$; (b) $4-\mathrm{NO}_{2} \mathrm{C}_{6} \mathrm{H}_{4}$; (c) 3- $\mathrm{NO}_{2} \mathrm{C}_{6} \mathrm{H}_{4}$; (d) $2-\mathrm{NO}_{2} \mathrm{C}_{6} \mathrm{H}_{4}$; (e) 4- $\mathrm{MeOC}_{6} \mathrm{H}_{4}$; (f) $3,4\left(\mathrm{OCH}_{3}\right)_{2} \mathrm{C}_{6} \mathrm{H}_{3} ;$ (g) $4-\mathrm{CH}_{3} \mathrm{C}_{6} \mathrm{H}_{4}$; (h) $4-\mathrm{ClC}_{6} \mathrm{H}_{4}$ (i) 2,4- $\mathrm{Cl}_{2} \mathrm{C}_{6} \mathrm{H}_{3}$; (j) 3- $\mathrm{BrC}_{6} \mathrm{H}_{4}$; (k) 3-PhCOO- $\mathrm{C}_{6} \mathrm{H}_{4}$; (l) $\mathrm{C}_{6} \mathrm{H}_{5} \mathrm{CH}=\mathrm{CH}$; (m) $\mathrm{CH}_{3} \mathrm{CH}=\mathrm{CH}$; (n) 2-Furyl; (o) 3-Pyridyl; (p) 2-Thienyl; (q) $\mathrm{CH}_{3}$; (r) $\mathrm{C}_{2} \mathrm{H}_{5}$; (s) $\mathrm{CH}_{3} \mathrm{CH}_{2} \mathrm{CH}_{2}$; (t) $\mathrm{CH}_{3} \mathrm{CH}_{2} \mathrm{CH}_{2} \mathrm{CH}_{2}$; (u) $\mathrm{C}_{6} \mathrm{H}_{13}$.

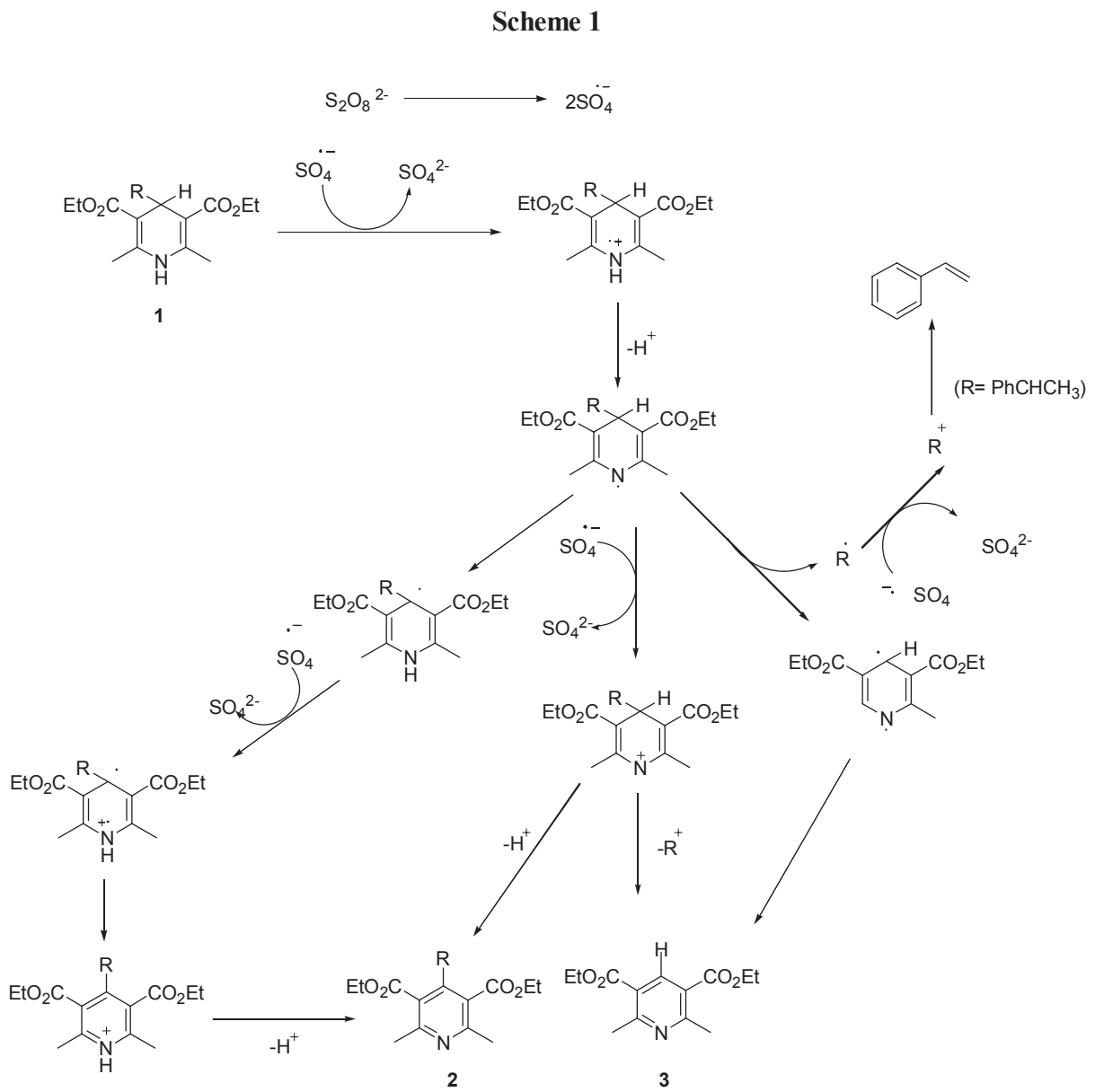

Scheme 2. Proposed mechanism for CTAPDS catalyzed oxidative aromatization of 1,4-DHPS

temperature but at reflux conditions it gave $100 \%$ conversion (TLC) within $10 \mathrm{~min}$. Acetonitrile was superior to other solvents as reaction failed to continue after some conversion in other solvents. The reactions were smooth and the effect of an electron donating or an electron-withdrawing group on the aromatic ring of the 1,4-DHP was not observed.

To establish the generality of the reagent CATPDS, various alkyl, aryl, and heterocyclic substituted Hantzsch 1,4-DHP were oxidized under the above cited conditions. The oxidation proceeded smoothly with 1,4-dihydropyridine substrates bearing substituents at the 4-position such as hydrogen, methyl, $n$-alkyl/ alkenyl, aryl and heterocyclic groups. However, in the case of oxidation of the 1,4-DHP with isopropyl/benzyl/cyclohexyl/ cyclohex-3-enyl/1-phenylethyl group at 4-position gave ex- clusively dealkylated/debenzylated pyridine derivatives (3) (Table 1). These substituents are debarred with the formation of dealkylated/debenzylated product either due to stability of radical cation formed during the reaction via single electron mechanism (SET) or electron donating ability of the corresponding radicals (Scheme 2). This mechanism is further confirmed by isolation of styrene (Table 1, Entry 26).

The application of peroxodisulphate ion with Co(II) or without $\mathrm{Co}$ (II) in the oxidative aromatization of 1,4-dihydropyridines has already been reported. ${ }^{13,38}$ To the best of our knowledge, cetyltrimethylammonium peroxodisulphate (CATPDS) is not used in oxidation of 1,4-DHP. Thus, we hoped that the CATPDS would be superior to the previously reported other peroxodisulphate oxidants because of i) less reaction time (ii) 
Table 1. Oxidative aromatization of 1,4-DHPs derivatives with cetyltrimethylammonium peroxodisulfate (1.2 equivalent)

\begin{tabular}{|c|c|c|c|c|c|}
\hline Entry & $\mathrm{R}$ & Product & $\begin{array}{c}\text { Reaction } \\
\text { Time } \\
\mathrm{t}(\mathrm{min})\end{array}$ & $\begin{array}{l}\text { Yield }^{a} \\
(\%)\end{array}$ & $\underset{\left({ }^{\circ} \mathrm{C}\right)}{\mathrm{mp}^{b}}$ \\
\hline 1 & $\mathrm{C}_{6} \mathrm{H}_{5}$ & $2 a$ & 6 & 97 & $62-63$ \\
\hline 2 & $4-\mathrm{NO}_{2} \mathrm{C}_{6} \mathrm{H}_{4}$ & $2 b$ & 7 & 95 & $112-113$ \\
\hline 3 & $3-\mathrm{NO}_{2} \mathrm{C}_{6} \mathrm{H}_{4}$ & $2 \mathrm{c}$ & 7 & 94 & $61-62$ \\
\hline 4 & $2-\mathrm{NO}_{2} \mathrm{C}_{6} \mathrm{H}_{4}$ & 2d & 7 & 94 & $73-75$ \\
\hline 5 & $4-\mathrm{MeOC}_{6} \mathrm{H}_{4}$ & $2 \mathrm{e}$ & 7 & 95 & $51-52$ \\
\hline 6 & $3,4\left(\mathrm{OCH}_{3}\right)_{2} \mathrm{C}_{6} \mathrm{H}_{3}$ & $2 f$ & 5 & 95 & $100-101$ \\
\hline 7 & $4-\mathrm{CH}_{3} \mathrm{C}_{6} \mathrm{H}_{4}$ & $2 \mathrm{~g}$ & 5 & 94 & $71-72$ \\
\hline 8 & $4-\mathrm{ClC}_{6} \mathrm{H}_{4}$ & $2 \mathrm{~h}$ & 7 & 95 & $69-71$ \\
\hline 9 & 2,4- $\mathrm{Cl}_{2} \mathrm{C}_{6} \mathrm{H}_{3}$ & $2 \mathbf{i}$ & 8 & 95 & $112-113$ \\
\hline 10 & $3-\mathrm{BrC}_{6} \mathrm{H}_{4}$ & $2 \mathrm{j}$ & 8 & 92 & $70-72$ \\
\hline 11 & 3-PhCOO- $\mathrm{C}_{6} \mathrm{H}_{4}$ & $2 \mathrm{k}$ & 10 & 90 & $260-261$ \\
\hline 12 & $\mathrm{C}_{6} \mathrm{H}_{5} \mathrm{CH}=\mathrm{CH}$ & 21 & 9 & 95 & $161-162$ \\
\hline 13 & $\mathrm{CH}_{3} \mathrm{CH}=\mathrm{CH}$ & $2 \mathrm{~m}$ & 8 & 92 & oil \\
\hline 14 & 2-Furyl & $2 n$ & 7 & 93 & oil \\
\hline 15 & 2-Pyridyl & 20 & 7 & 94 & $90-92$ \\
\hline 16 & 2-Thienyl & $2 p$ & 7 & 91 & $78-79$ \\
\hline 17 & $\mathrm{CH}_{3}$ & $2 q$ & 5 & 94 & oil \\
\hline 18 & $\mathrm{C}_{2} \mathrm{H}_{5}$ & $2 r$ & 5 & 95 & oil \\
\hline 19 & $\mathrm{CH}_{3} \mathrm{CH}_{2} \mathrm{CH}_{2}$ & $2 s$ & 5 & 95 & oil \\
\hline 20 & $\mathrm{CH}_{3} \mathrm{CH}_{2} \mathrm{CH}_{2} \mathrm{CH}_{2}$ & $2 t$ & 6 & 95 & oil \\
\hline 21 & $\mathrm{C}_{6} \mathrm{H}_{13}$ & $2 u$ & 6 & 92 & oil \\
\hline 22 & $\left(\mathrm{CH}_{3}\right)_{2} \mathrm{CH}$ & 3 & 5 & 94 & $70-71$ \\
\hline 23 & $\mathrm{C}_{6} \mathrm{H}_{5} \mathrm{CH}_{2}$ & 3 & 6 & 95 & $70-71$ \\
\hline 24 & $\mathrm{C}_{6} \mathrm{H}_{11}$ (cyclohexyl) & 3 & 10 & 96 & $70-71$ \\
\hline 25 & $\mathrm{C}_{6} \mathrm{H}_{9}$ (cyclohex-3-enyl) & 3 & 10 & 96 & $70-71$ \\
\hline 26 & $\mathrm{C}_{6} \mathrm{H}_{5}-\mathrm{CH}-\mathrm{CH}_{3}$ & 3 & 5 & 95 & $70-71$ \\
\hline 27 & $\mathrm{H}$ & 3 & 3 & 93 & $70-71$ \\
\hline
\end{tabular}

${ }^{\bar{a}}$ Yields are isolated. ${ }^{b}$ Melting points are uncorrected and compared with literature reports.

high yield (iii) no dealkylation of 4-n-alkyl/alkenyl substituents of 1,4-DHP i.e., selective oxidation (iv) avoidance of toxicity of many transition metal involved in such process (v) solubility in most organic solvent (vi) commercial availability.

\section{Experimental Section}

All chemicals used in this study were purchased from local venders and used without further purification. Melting points were determined on a buchi oil heating melting apparatus and are uncorrected. ${ }^{1} \mathrm{H}$ NMR spectra were recorded in $\mathrm{CDCl}_{3}$ on Brucker- $300 \mathrm{~Hz}$ spectrometer using TMS as internal standard (chemical shift in $\delta, \mathrm{ppm}$ ). IR spectra were taken on a Perkin Elmer 1600, FTIR spectrophotometer using KBr pellets and peaks are reported in $\mathrm{cm}^{-1}$.

1,4-Dihydropyridine were prepared according to described procedures. $^{30-35}$

Preparation of cetyltrimethylammonium peroxodisufate. Potassium peroxodisulphate $(0.01 \mathrm{~mol})$ in $10 \mathrm{~mL}$ water was added slowly to an aqueous solution of cetyltrimethylammonium bromide $(0.025 \mathrm{~mol})$ with continuous stirring on a magnetic stirrer. A light yellow coloured compound appeared slowly. Stirring was continued for $30 \mathrm{~min}$. The resulting light yellow coloured compound was filtered, washed with water for several times until no trace of bromide ion was detected in the filtrate. It was vacuum dried and kept in a dark bottle inside desiccator, yield $93 \%$. ${ }^{1} \mathrm{H}-\mathrm{NMR}\left(\mathrm{CDCl}_{3}\right) \delta 0.88(\mathrm{t}, J=7.6 \mathrm{~Hz}, 6 \mathrm{H}) ; 1.25$ 1.32 (multiplet, $52 \mathrm{H}$ ); 1.81 (multiplet, $4 \mathrm{H}$ ), 3.38-3.46 (multiplet, 22H). $m / z$ 760.57. Anal. Calcd. for $\mathrm{C}_{38} \mathrm{H}_{84} \mathrm{~N}_{2} \mathrm{O}_{8} \mathrm{~S}_{2}$ : C, 59.96; $\mathrm{H}$, 11.12 ; N, 3.68. Found: C, 60.17; H, 11.23; N, 3.78\%.

General procedure for oxidation of Hantzsch 1,4-DHPs. In a $50 \mathrm{~mL}$ round bottom flask, cetyltrimethylammonium peroxodisulfate (1.2 mmol), Hantzsch-1,4-dihydropyridime (1.0 mmol) in $20 \mathrm{~mL}$ acetonitrile was added. The reaction mixture was refluxed on water bath for the time indicated in Table 1 . The progress of reaction was monitored by TLC (hexane/ethylacetate $=9 / 1)$. After completion of reaction, solvent was evaporated and the crude reaction mixture was purified by column chromatography using ethyl acetate-hexane (1:8).

The characterization and spectral data for selected products.

Diethyl-4-phenyl-2,6-dimethylpyridine-3,5-dicarboxylate (2a): IR (KBr) 3014, 2986, 1723, 1591, 1498, 1302, 1250, 1170 , $791,760 \mathrm{~cm}^{-1} .{ }^{1} \mathrm{H}-\mathrm{NMR}\left(\mathrm{CDCl}_{3}\right) \delta 1.23(\mathrm{t}, J=7.12 \mathrm{~Hz}, 6 \mathrm{H}$, $\left.\mathrm{CH}_{3}\right), 4.26\left(\mathrm{q}, J=7.12 \mathrm{~Hz}, 4 \mathrm{H}, \mathrm{OCH}_{2}\right), 2.65\left(\mathrm{~s}, 6 \mathrm{H}, \mathrm{CH}_{3}\right), 7.18$ (m, 2H), $7.30(\mathrm{~m}, 3 \mathrm{H})$. Anal. Calcd. for $\mathrm{C}_{19} \mathrm{H}_{21} \mathrm{NO}_{4}$ : C, 69.71; H, 6.47; N, 4.28. Found: C, 69.83; H, 6.38; N, 4.32.

Diethyl-4-(4-nitrophenyl)-2,6-dimethylpyridine-3,5-dicarboxylate (2b): IR (KBr) 3012, 2977, 1723, 1557, 1518, 1349 , $1116,865,843,745 \mathrm{~cm}^{-1} .{ }^{1} \mathrm{H}-\mathrm{NMR}\left(\mathrm{CDCl}_{3}\right) \delta 1.20(\mathrm{t}, J=7.10$ $\left.\mathrm{Hz}, 6 \mathrm{H}, \mathrm{CH}_{3}\right), 2.69$ (s, 6H, $\left.\mathrm{CH}_{3}\right), 4.27$ (q, $J=7.10 \mathrm{~Hz}, 4 \mathrm{H}$, $\left.\mathrm{OCH}_{2}\right), 7.41(\mathrm{~d}, J=8.2 \mathrm{~Hz}, 2 \mathrm{H})-8.22(\mathrm{~d}, J=8.2 \mathrm{~Hz}, 2 \mathrm{H})$. Anal. Calcd. for $\mathrm{C}_{19} \mathrm{H}_{20} \mathrm{~N}_{2} \mathrm{O}_{6}$ : C, 61.29; H, 5.41; N, 7.53. Found: $\mathrm{C}$, $61.31 ; \mathrm{H}, 5.36 ; \mathrm{N}, 7.50$.

Diethyl-4-(3-nitrophenyl)-2,6-dimethylpyridine-3,5-dicarboxylate (2c): IR (KBr) 3015, 2980, 1716, 1590, 1555, 1520, $1358,1280,1183,870,785,715 \mathrm{~cm}^{-1} .{ }^{1} \mathrm{H}-\mathrm{NMR}\left(\mathrm{CDCl}_{3}\right) \delta 1.21$ $\left(\mathrm{t}, J=7.11 \mathrm{~Hz}, 6 \mathrm{H}, \mathrm{CH}_{3}\right), 2.70\left(\mathrm{~s}, 6 \mathrm{H}, \mathrm{CH}_{3}\right), 4.25(\mathrm{q}, J=7.11 \mathrm{~Hz}$, $\left.4 \mathrm{H}, \mathrm{OCH}_{2}\right), 7.58-8.28(\mathrm{~m}, 4 \mathrm{H})$. Anal. Calcd. for $\mathrm{C}_{19} \mathrm{H}_{20} \mathrm{~N}_{2} \mathrm{O}_{6}$ : C, 61.29; H, 5.41; N, 7.53. Found: C, 61.15; H, 5.49; N, 7.33.

Diethyl-4-(2-nitrophenyl)-2,6-dimethylpyridine-3,5-dicarboxylate (2d): IR (KBr) 3005, 2983, 1725, 1605, 1548, 1512, $1358,1278,1191,762,700 \mathrm{~cm}^{-1} .{ }^{1} \mathrm{H}-\mathrm{NMR}\left(\mathrm{CDCl}_{3}\right) \delta 1.19(\mathrm{t}$, $\left.J=7.11 \mathrm{~Hz}, 6 \mathrm{H}, \mathrm{CH}_{3}\right), 2.70\left(\mathrm{~s}, 6 \mathrm{H}, \mathrm{CH}_{3}\right), 4.28(\mathrm{q}, J=7.11 \mathrm{~Hz}$, $\left.4 \mathrm{H}, \mathrm{OCH}_{2}\right), 7.48-8.25(\mathrm{~m}, 4 \mathrm{H})$. Anal. Calcd. for $\mathrm{C}_{19} \mathrm{H}_{20} \mathrm{~N}_{2} \mathrm{O}_{6}$ : C, 61.29; H, 5.41; N, 7.53. Found: C, 61.08; H, 5.22; N, 7.63.

Diethyl-4-(4-methoxyphenyl)-2,6-dimethylpyridine-3,5dicarboxylate (2e): IR (KBr) 3030, 2973, 1729, 1614, 1557, $1291,1107,857,835,779 \mathrm{~cm}^{-1} .{ }^{1} \mathrm{H}-\mathrm{NMR}\left(\mathrm{CDCl}_{3}\right) \delta 1.20(\mathrm{t}, J=$ $\left.7.12 \mathrm{~Hz}, 6 \mathrm{H}, \mathrm{CH}_{3}\right), 4.25\left(\mathrm{q}, J=7.12 \mathrm{~Hz}, 4 \mathrm{H}, \mathrm{OCH}_{2}\right), 2.66(\mathrm{~s}$, $\left.6 \mathrm{H}, \mathrm{CH}_{3}\right), 3.82\left(\mathrm{~s}, 3 \mathrm{H}, \mathrm{OCH}_{3}\right), 6.89(\mathrm{~d}, J=8.6 \mathrm{~Hz}, 2 \mathrm{H}), 7.10$ $(\mathrm{d}, J=8.6 \mathrm{~Hz}, 2 \mathrm{H})$. Anal. Calcd. for $\mathrm{C}_{20} \mathrm{H}_{23} \mathrm{NO}_{5}$ : C, 67.21; $\mathrm{H}$, 6.49 ; N, 3.92. Found: C, 67.05; H, 6.40; N, 3.88.

Diethyl-4-(3,4-dimethoxyphenyl)-2,6-dimethylpyridine3,5-dicarboxylate (2f): IR (KBr) 3030, 2973, 1729, 1614, 1557 , $1291,1107,857,835,779 \mathrm{~cm}^{-1} .{ }^{1} \mathrm{H}-\mathrm{NMR}\left(\mathrm{CDCl}_{3}\right) \delta 1.20(\mathrm{t}, J=$ $\left.7.12 \mathrm{~Hz}, 6 \mathrm{H}, \mathrm{CH}_{3}\right), 4.25$ (q, $\left.J=7.12 \mathrm{~Hz}, 4 \mathrm{H}, \mathrm{OCH}_{2}\right), 2.66$ (s, $6 \mathrm{H}$, $\left.\mathrm{CH}_{3}\right), 3.86\left(\mathrm{~s}, 6 \mathrm{H}, \mathrm{OCH}_{3}\right), 6.95(\mathrm{~m}, 2 \mathrm{H}), 7.21(\mathrm{~m}, 1 \mathrm{H})$. Anal. Calcd. for $\mathrm{C}_{21} \mathrm{H}_{25} \mathrm{NO}_{6}$ : C, 65.10; $\mathrm{H}, 6.50 ; \mathrm{N}, 3.62$. Found: $\mathrm{C}$, $65.25 ; \mathrm{H}, 6.61 ; \mathrm{N}, 3.77$.

Diethyl-4-(4-methylphenyl)-2,6-dimethylpyridine-3,5-dicarboxylate (2g): IR (KBr) 3013, 2983, 1727, 1571, 1446, 
$1239,1033,821,856,775 \mathrm{~cm}^{-1} .{ }^{1} \mathrm{H}-\mathrm{NMR}\left(\mathrm{CDCl}_{3}\right) \delta 1.23(\mathrm{t}, J=$ $\left.7.12 \mathrm{~Hz}, 6 \mathrm{H}, \mathrm{CH}_{3}\right), 2.37$ (s, 3H, $\left.\mathrm{CH}_{3}\right), 2.64\left(\mathrm{~s}, 6 \mathrm{H}, \mathrm{CH}_{3}\right), 4.29$ $\left(\mathrm{q}, J=7.12 \mathrm{~Hz}, 4 \mathrm{H}, \mathrm{OCH}_{2}\right), 7.11(\mathrm{~d}, J=6.8 \mathrm{~Hz}, 2 \mathrm{H}), 7.21(\mathrm{~d}$, $J=6.8 \mathrm{~Hz}, 2 \mathrm{H})$. Anal. Calcd. for $\mathrm{C}_{20} \mathrm{H}_{23} \mathrm{NO}_{4}: \mathrm{C}, 70.36 ; \mathrm{H}, 6.79$; N, 4.10. Found: C, 70.23; H, 6.56; N, 4.33.

Diethyl-4-(4-chlorophenyl)-2,6-dimethylpyridine-3,5-dicarboxylate (2h): IR (KBr) 3025, 2984, 1729, 1580, 1231, 1104 , $1044,858,658 \mathrm{~cm}^{-1}$. ${ }^{1} \mathrm{H}-\mathrm{NMR}\left(\mathrm{CDCl}_{3}\right) \delta 1.24(\mathrm{t}, J=7.12 \mathrm{~Hz}$, $\left.6 \mathrm{H}, \mathrm{CH}_{3}\right), 4.27$ (q, J=7.12 Hz, 4H, $\left.\mathrm{OCH}_{2}\right), 2.69\left(\mathrm{~s}, 6 \mathrm{H}, \mathrm{CH}_{3}\right)$, $7.13(\mathrm{~d}, J=9.01 \mathrm{~Hz}, 2 \mathrm{H}), 7.32$ (d, $J=9.01 \mathrm{~Hz}, 2 \mathrm{H})$. Anal. Calcd. for $\mathrm{C}_{19} \mathrm{H}_{20} \mathrm{ClNO}_{4}$ : C, 63.07; H, 5.57; N, 3.87. Found: C, 62.97; H, 5.44; N, 4.03.

Diethyl-4-(2,4-dichlorophenyl)-2,6-dimethylpyridine-3,5dicarboxylate (2i): IR (KBr) 3008, 2986, 1730, 1560, 1480, $1280,1228,1108,856,775,700 \mathrm{~cm}^{-1} .{ }^{1} \mathrm{H}-\mathrm{NMR}\left(\mathrm{CDCl}_{3}\right) \delta 1.23$ (t, $\left.J=7.13 \mathrm{~Hz}, 6 \mathrm{H}, \mathrm{CH}_{3}\right), 4.33$ (q, $\left.J=7.13 \mathrm{~Hz}, 4 \mathrm{H}, \mathrm{OCH}_{2}\right), 2.67$ (s, $\left.6 \mathrm{H}, \mathrm{CH}_{3}\right), 7.15-7.42(\mathrm{~m}, 3 \mathrm{H})$. Anal. Calcd. for $\mathrm{C}_{19} \mathrm{H}_{19} \mathrm{Cl}_{2} \mathrm{NO}_{4}$ : C, 57.59; H, 4.83; N, 3.53. Found: C, 57.77; H, 5.00; N, 3.49.

Diethyl-4-(3-bromophenyl)-2,6-dimethylpyridine-3,5-dicarboxylate (2j): IR (KBr) 3026, 2986, 1726, 1561, 1278, 1230, $1108,1035,865,787,698 \mathrm{~cm}^{-1} .{ }^{1} \mathrm{H}-\mathrm{NMR}\left(\mathrm{CDCl}_{3}\right) \delta 1.22(\mathrm{t}, J=$ $\left.7.11 \mathrm{~Hz}, 6 \mathrm{H}, \mathrm{CH}_{3}\right), 4.31$ (q, $\left.J=7.11 \mathrm{~Hz}, 4 \mathrm{H}, \mathrm{OCH}_{2}\right), 2.66(\mathrm{~s}, 6 \mathrm{H}$, $\left.\mathrm{CH}_{3}\right)$, 7.20-7.44 (m, $\left.4 \mathrm{H}\right)$. Anal. Calcd. for $\mathrm{C}_{19} \mathrm{H}_{20} \mathrm{BrNO}_{4}$ : C, 56.17; H, 4.96; N, 3.45. Found: C, 56.30; H, 5.10; N, 3.27.

Diethyl 4-(3-(benzoyloxy)phenyl)-2,6-dimethylpyridine3,5-dicarboxylate (2k): IR (KBr) 3014, 2986, 1735, 1723, 1574, $1498,1380,1250,1170,791,760 \mathrm{~cm}^{-1} .{ }^{1} \mathrm{H}-\mathrm{NMR}\left(\mathrm{CDCl}_{3}\right) \delta 1.24$ (t, $\left.J=7.11 \mathrm{~Hz}, 6 \mathrm{H}, \mathrm{CH}_{3}\right), 4.26\left(\mathrm{q}, J=7.11 \mathrm{~Hz}, 4 \mathrm{H}, \mathrm{OCH}_{2}\right)$, $2.63\left(\mathrm{~s}, 6 \mathrm{H}, \mathrm{CH}_{3}\right), 7.33-7.77(\mathrm{~m}, 7 \mathrm{H}), 8.20(\mathrm{~m}, 2 \mathrm{H})$. Anal. Calcd. for $\mathrm{C}_{26} \mathrm{H}_{25} \mathrm{NO}_{6}$ : C, 69.79; H, 5.63; N, 3.13. Found: C, 69.83; $\mathrm{H}, 6.38 ; \mathrm{N}, 4.32$.

Diethyl 2,6-dimethyl-4-cinnamylpyridine-3,5-dicarboxylate (2I): IR (KBr) 3067, 2966, 2835, 1729, 1588, 1552, 1466, $1240,1230,1108,1035,962,835,739 \mathrm{~cm}^{-1} .{ }^{1} \mathrm{H}-\mathrm{NMR}\left(\mathrm{CDCl}_{3}\right)$ $\delta 1.20\left(\mathrm{t}, J=7.13 \mathrm{~Hz}, 6 \mathrm{H}, \mathrm{CH}_{3}\right), 4.29\left(\mathrm{q}, J=7.13 \mathrm{~Hz}, 4 \mathrm{H}, \mathrm{OCH}_{2}\right)$, $2.64\left(\mathrm{~s}, 6 \mathrm{H}, \mathrm{CH}_{3}\right), 6.65(\mathrm{~d}, J=15.3 \mathrm{~Hz}, 1 \mathrm{H}) ; 7.18(\mathrm{~d}, J=15.3$ $\mathrm{Hz}, 1 \mathrm{H})$; 7.30-7.60 (m, 5H). Anal. Calcd. for $\mathrm{C}_{21} \mathrm{H}_{23} \mathrm{NO}_{4}$ : C, 71.37; H, 6.56; N, 3.96. Found: C, 71.41; H, 6.66; N, 4.11.

Diethyl 2,6-dimethyl-4-(prop-1-enyl)pyridine-3,5-dicarboxylate (2m): IR (KBr) 2978, 2834, 1721, 1588, 1566, 1466, $1245,1230,1108,861 \mathrm{~cm}^{-1} .{ }^{1} \mathrm{H}-\mathrm{NMR}\left(\mathrm{CDCl}_{3}\right) \delta 1.78(\mathrm{t}, J=$ $6.8 \mathrm{~Hz}, 3 \mathrm{H}) ; 1.22$ (t, $\left.J=7.11 \mathrm{~Hz}, 6 \mathrm{H}, \mathrm{CH}_{3}\right), 4.27$ (q, $J=7.11$ $\left.\mathrm{Hz}, 4 \mathrm{H}, \mathrm{OCH}_{2}\right), 2.62\left(\mathrm{~s}, 6 \mathrm{H}, \mathrm{CH}_{3}\right), 6.01-6.12(\mathrm{~m}, 1 \mathrm{H}) ; 6.42$ (d, $J=16.1 \mathrm{~Hz}, 1 \mathrm{H})$. Anal. Calcd. for $\mathrm{C}_{16} \mathrm{H}_{21} \mathrm{NO}_{4}$ : C, 65.96; $\mathrm{H}, 7.27$; N, 4.81. Found: C, 66.11; H, 7.33; N, 5.00.

Diethyl 4-(2-thienyl)-2,6-dimethyl-3,5-pyridinedicarboxylate (2p): $\mathrm{IR}(\mathrm{KBr}) 3101,2972,2827,1722,1566,1466,1242$, $1102,1022,860,710 \mathrm{~cm}^{-1} .{ }^{1} \mathrm{H}-\mathrm{NMR}\left(\mathrm{CDCl}_{3}\right) \delta 1.11(\mathrm{t}, J=7.11$ $\mathrm{Hz}, 6 \mathrm{H}), 2.44$ (s, 6H), 4.18 (q, $J=7.11 \mathrm{~Hz}, 4 \mathrm{H}), 7.17$ (m, 1H), 7.40-7.50 (m, 2H).

Diethyl 4-(2-pyridyl)-2,6-dimethyl-3,5-pyridinedicarboxylate (2o): $\mathrm{IR}(\mathrm{KBr}) 3111,2991,2855,1729,1611,1522,1457$, $1241,1111,859,710 \mathrm{~cm}^{-1} .{ }^{1} \mathrm{H}-\mathrm{NMR}\left(\mathrm{CDCl}_{3}\right) \delta 1.15(\mathrm{t}, J=$ $7.12 \mathrm{~Hz}, 6 \mathrm{H}), 2.62(\mathrm{~s}, 6 \mathrm{H}), 4.15(\mathrm{q}, J=7.4 \mathrm{~Hz}, 4 \mathrm{H}), 7.0-7.8$ (m, $3 \mathrm{H}), 8.51(\mathrm{~d}, J=5.2 \mathrm{~Hz}, 1 \mathrm{H})$.

Diethyl 4-(2-furyl)-2,6-dimethyl-3,5-pyridinedicarboxylate (2p): IR (KBr) 3121, 2988, 2840, 1727, 1571, 1241, 1109, 755 $\mathrm{cm}^{-1} .{ }^{1} \mathrm{H}-\mathrm{NMR}\left(\mathrm{CDCl}_{3}\right) \delta 1.18(\mathrm{t}, J=7.0 \mathrm{~Hz}, 6 \mathrm{H}), 2.59(\mathrm{~s}$,
$6 \mathrm{H}), 4.21$ (q, $J=7.0 \mathrm{~Hz}, 4 \mathrm{H},), 6.56-6.66(\mathrm{~m}, 2 \mathrm{H}), 7.42(\mathrm{~m}, 1 \mathrm{H})$.

Diethyl 2,6-dimethyl-4-methylpyridine-3,5-dicarboxylate (2q): IR (KBr) 2981, 2870, 1726, 1568, 1446, 1285, 1220, $1106,1045,871,777 \mathrm{~cm}^{-1} .{ }^{1} \mathrm{H}-\mathrm{NMR}\left(\mathrm{CDCl}_{3}\right) \delta 1.23(\mathrm{t}, J=7.10$ $\left.\mathrm{Hz}, 6 \mathrm{H}, \mathrm{CH}_{3}\right), 2.19$ (s, 3H, $\left.\mathrm{CH}_{3}\right), 2.51\left(\mathrm{~s}, 6 \mathrm{H}, \mathrm{CH}_{3}\right), 4.25$ (q, $J=$ 7.10 Hz, $4 \mathrm{H}, \mathrm{OCH}_{3}$ ). Anal. Calcd. for $\mathrm{C}_{14} \mathrm{H}_{19} \mathrm{NO}_{4}$ : C, 63.38; $\mathrm{H}$, 7.22; N, 5.28. Found: C, 63.23; H, 7.32; N, 5.01.

Diethyl 2,6-dimethyl-4-ethylpyridine-3,5-dicarboxylate (2r): IR (KBr) 2992, 2879, 1731, 1576, 1438, 1286, 1112, 1045, 923, $847,751 \mathrm{~cm}^{-1} .{ }^{1} \mathrm{H}-\mathrm{NMR}\left(\mathrm{CDCl}_{3}\right) \delta 1.08\left(\mathrm{t}, J=7.5 \mathrm{~Hz}, 3 \mathrm{H}, \mathrm{CH}_{3}\right)$, $1.25\left(\mathrm{t}, J=7.11 \mathrm{~Hz}, 6 \mathrm{H}, \mathrm{CH}_{3}\right), 2.49\left(\mathrm{~s}, 6 \mathrm{H}, \mathrm{CH}_{3}\right), 2.78(\mathrm{q}, J=7.5$ $\left.\mathrm{Hz}, 2 \mathrm{H}, \mathrm{CH}_{2}\right), 4.25$ (q, J=7.11 Hz, 4H, $\left.\mathrm{OCH}_{3}\right)$. Anal. Calcd. for $\mathrm{C}_{15} \mathrm{H}_{21} \mathrm{NO}_{4}$ : C, 64.50; H, 7.58; N, 5.01. Found: C, 64.78; H, 7.78; N, 4.94 .

Diethyl 2,6-dimethyl-4-n-hexylpyridine-3,5-dicarboxylate (2u): IR (KBr) 2989, 2879, 1726, 1569, 1445, 1222, 1102, 1044, $917,756 \mathrm{~cm}^{-1} .{ }^{1} \mathrm{H}-\mathrm{NMR}\left(\mathrm{CDCl}_{3}\right) \delta 1.08(\mathrm{t}, J=6.6 \mathrm{~Hz}, 3 \mathrm{H}), 1.24$ $\left(\mathrm{t}, J=7.10 \mathrm{~Hz}, 6 \mathrm{H}, \mathrm{CH}_{3}\right), 1.32-1.42(\mathrm{~m}, 8 \mathrm{H}) ; 1.56(\mathrm{t}, J=6.6$ $\mathrm{Hz}, 2 \mathrm{H}) ; 2.48$ (s, 6H, CH $), 4.22$ (q, $\left.J=7.10 \mathrm{~Hz}, 4 \mathrm{H}, \mathrm{OCH}_{3}\right)$. Anal. Calcd. for $\mathrm{C}_{19} \mathrm{H}_{29} \mathrm{NO}_{4}$ : C, 68.03; H, 8.71; N, 4.18. Found: C, 68.19; H, 8.78; N, 4.23.

Diethyl 2,6-dimethylpyridine-3,5-dicarboxylate (3): IR (KBr) 2974, 1721, 1588, 1555, 1298, 1254, 1123, 1022, 777 $\mathrm{cm}^{-1} .{ }^{1} \mathrm{H}-\mathrm{NMR}\left(\mathrm{CDCl}_{3}\right) \delta 1.35\left(\mathrm{t}, J=7.11 \mathrm{~Hz}, 6 \mathrm{H}, \mathrm{CH}_{3}\right), 2.74$ (s, 6H, $\left.\mathrm{CH}_{3}\right), 4.28$ (q, $\left.J=7.11 \mathrm{~Hz}, 4 \mathrm{H}, \mathrm{OCH}_{3}\right)$. Anal. Calcd. for $\mathrm{C}_{13} \mathrm{H}_{17} \mathrm{NO}_{4}$ : C, 62.14; H, 6.82; N, 5.57. Found: C, 61.92; H, 7.02; N, 5.44 .

Acknowledgments. We are thankful to authorities of GNKCYamuna Nagar and GJUST-Hissar for providing research facilities.

\section{References}

1. (a) Boger, D. L.; Nakahara, S. J. Org. Chem. 1991, 56, 880. (b) Boger, D. L.; Kasper, A. M. J. Am. Chem. Soc. 1989, 111, 1517. (c) Zhang, T. Y.; Stout, J. R.; Keay, J. G.; Scriven, E. F. V.; Toomey, J. E.; Goe, G. L. Tetrahedron 1995, 51, 13177. (d) Ma, X.; Gang, D. R. Nat. Prod. Rep. 2004, 21, 752.

2. (a) Fletcher, M. D.; Hurst, T. E.; Miles, T. J.; Moody, C. J. Tetrahedron 2006, 62, 5454. (b) Evdokimov, N. M.; Magedov, I. V.; Kireev, A. S.; Kornienko, A. Org. Lett. 2006, 8, 899. (c) Movassaghi, M.; Hill, M. D. J. Am. Chem. Soc. 2006, 128, 4592. (d) Winter, A.; Risch, N. Synthesis 2003, 2667. (e) Thomas, A. D.; Asokan, C. V. Tetrahedron Lett. 2002, 43, 2273. (f) Tanaka, K.; Mori, H.; Yamamoto, M.; Katsumara, S. J. Org. Chem. 2001, 66, 3099. (g) Mashraqui, S. H.; Karnik, M. A. Tetrahedron Lett. 1998, 39, 4895. (h) Singh, K.; Singh, J.; Singh, H. Tetrahedron 1998, 54, 935. (i) Renslo, A. R.; Danheiser, R. L. J. Org. Chem. 1998, 63, 7840. (j) Vijn, R. J.; Arts, H. J.; Green, R.; Castelijns, A. M. Synthesis 1994, 573. (k) Ahmed, S.; Baruah, R. C. Tetrahedron Lett. 1996, 37, 8231. (1) Komatsu, M.; Ohgishi, H.; Takamatsu, S.; Ohshiro, Y.; Agawa, T. Angew. Chem., Int. Ed. Engl. 1982, 21, 213. (m) Van Aken, K. J.; Lux, G. M.; Deroover, G. G.; Meerpoel, L.; Hoornaert, G. J. Tetrahedron 1994, 50, 5211. (n) Anabha, E. R.; Nirmala, K. N.; Thomas, A.; Asokan, C. V. Synthesis 2007, 428.

3. (a) Shan, R.; Howlett, S. E.; Knaus, E. E. J. Med. Chem. 2002, 45, 955. (b) Perez-Reyes, E.; Schneider, T. Drug Dev. Res. 1994, 33, 295. (c) Triggle, D. J. Chirality 1996, 8, 35 and references therein.

4. (a) Flaim, S. F.; Zelis, R. Fed. Proc. 1981, 40, 2877. (b) Brewster, M. E.; Simay, A.; Czako, K.; Winwood, D.; Fatag, H.; Bodor, N. J. Org. Chem. 1989, 54, 3721. (c) Friedols, F.; Knox, R. J. Biochem. 
Pharmacol. 1992, 44, 631. (d) Janis, R. A.; Triggle, D. J. J. Med. Chem. 1983, 25, 775. (d) Mason, R. P.; Mak, I. T.; Trumbore, M. W.; Mason, P. E. Am. J. Cardiol. 1999, 84, 16. (e) Peri, R.; Padmanabhan, S.; Rutledge, A.; Singh, S.; Triggle, Da. J. J. Med. Chem. 2000, 43, 2906. (f) Khadikar, B.; Borkat, S. Synth. Commun. 1998, 207.

5. Mao, Y. Z.; Jin, M. Z.; Liu, Z. L.; Wu, L. M. Org. Lett. 2000, $2,741$.

6. Zolfigol, M. A.; Zebarjadian, M. H.; Sadeghi, M. M.; Mohammadpoor-Baltork, I.; Memarian, H. R.; Shamsipur, M. Synth. Commun. 2001, 31, 929 .

7. Memarian, H. R.; Sadeghi, M. M.; Aliyan, H. Indian J. Chem. 1998, 37B, 219.

8. Sabitha, G.; Reddy, G. S. K.; Reddy, Ch. S.; Fatima, N.; Yadav, J. S. Synthesis 2003, 1267.

9. Wang, B.; Hu, Y.; Hu, H. Synth. Commun. 1999, $29,4193$.

10. Sadeghi, M. M.; Mohammadpoor-Baltork, I.; Memarian, H. R.; Sobhani, S. Synth. Commun. 2000, 30, 1661.

11. Guengerich, F. P.; Bocker, R. H. J. Biol. Chem. 1998, $263,8168$.

12. Pragst, F.; Kaltofen, B.; Volke, J.; Kuthan, J. J. Electroanal. Chem. 1981, 119, 301.

13. Anniyappam, M.; Muralidharan, D.; Perumal, P. T. Tetrahedron 2002, 58, 5069 .

14. Varma, R. S.; Kumar, D. Tetrahedron Lett. 1999, 40, 21.

15. Litvic, M. F.; Litvic, M.; Vinkovic, V. Tetrahedron 2008, 64, 10912 and references cited their in.

16. Esfahani, M. N.; Moghadam, M.; Tangestaninejad, S.; Mirkhani, V. Bio. Med. Chem. Lett. 2005, 15, 3276.

17. Sharma, S. D.; Hazarika, P.; Konwar, D. Catal. Commun. 2008, 9, 709 .

18. Adibia, H.; Hajipour, A. R. Bio. Med. Chem. Lett. 2007, 17, 1008.

19. Moghadam, M.; Nasr-Esfahani, M.; Tangestaninejad, S.; Mirkhani, V. Bio. Med. Chem. Lett. 2006, 16, 2026.

20. Filipan-Litvic, M.; Litvic, M.; Vinkovic, V. Tetrahedron 2008, 64, 5649.

21. Heravi, M. M.; Behbahani, F. K.; Oskooie, H. A.; Shoar, R. H. Tetrahedron Lett. 2005, 46, 2775.

22. Heravi, M. M.; Derikvand, F.; Hassan-Pour, S.; Bakhtiari, K.; Bamoharram, F. F.; Oskooie, H. A. Bio. Med. Chem. Lett. 2007, 17, 3305.

23. Eynde, J. J. V.; Mayence, A.; Maquestiau, A. Tetrahedron 1992 ,
48,463

24. Khalilzadeh, M. A.; Hosseini, A.; Sadeghifar, H.; Valipour, P. Acta Chim. Slov. 2007, 54, 900.

25. Zeynizadeh, B.; Dilmaghani, K. A.; Roozijoy, A. J. Chinese Chem. Soc. 2005, 52, 1001

26. Zolfigol, M. A.; Shirini, F.; Choghamarania, A. G.; Baltorkc, I. M. Green Chemistry 2002, 4, 562.

27. Zeynizadeh, B.; Dilmaghani, K. A.; Roozijoy, A. Synth. Commun. 2005, 35, 557 .

28. Zolfigol, M. A.; Salehi, P.; Choghamarani, A. G.; Safaiee, M.; Shahamirian, M. Synth. Commun. 2007, 37, 1817.

29. Bagley, M. C.; Lubinu, M. C. Synthesis 2006, 1283 and references cited therein.

30. Yadav, J. S.; Reddy, B. V. S.; Basak, A. K.; Baishya, G.; Narsaiah, A. V. Synthesis 2006, 451 and references cited therein.

31. Varma, R. S.; Kumar, D. J. Chem. Soc., Perkin Trans. 1 1999, 1755 and references cited therein.

32. Lee, K. H.; Ko, K. Y. Bull. Korean Chem. Soc. 2002, 23(11), 1505 and references cited therein

33. Cheng, D. P.; Chen, Z. C. Synth. Commun. 2002, 32(5), 793.

34. Kumar, P. Chinese J. Chemistry 2009, 27, 1487.

35. Kumar, P. J. Heterocyclic Chem. MS No JHET-10-0019, In Press.

36. (a) House, D. A. Chem. Rev. 1962, 62, 185. (b) Moschel, R. C.; Behrman, E. J. J. Org. Chem. 1974, 39, 1983. (c) Clerici, A.; Porta, O. J. Chem. Soc. Perkin Trans. 2 1980, 1234. (e) Fristad, W. E.; Peterson, J. R. Tetrahedron Lett. 1983, 24, 4547. (f) Nikishin, G. I.; Troyansky, E. I.; Lazareva, M. I. Tetrahedron Lett. 1984, 25, 4987. (g) Itahara, T.; Fujii, Y.; Tada, M. J. Org. Chem. 1988, 53, 3421 and references cited therein. (h) Yamasaki, S. Chem. Lett. 1992, 323. (i) Memarian, H. R.; Farhadi, A. J. Iran. Chem. Soc. 2009, 6(3), 638-646 and references cited therein. (j) Yang, S. G.; Hwang, J. P.; Park, M. Y.; Lee, K.; Kim, Y. H. Tetrahedron 2007, $63,5184$.

37. (a) Gratzel, C. K.; Jirousek, M.; Gratzel, M. Colloids Surf. 1985, 13, 221. (b) Gratzel, C. K.; Jirousek, M.; Gratzel, M. J. Phys. Chem. 1984, 88, 1055. (c) Norouzi, M.; Tajbakhsh, M.; Alinezhad, H.; Urimi, A. G. J. Chin. Chem. Soc. 2008, 55, 508. (d) Urimi, A. G.; Alinezhad, H.; Tajbakhsh, M. Acta Chim. Slov. 2008, 55, 481.

38. Memarian, H. R.; Mohammadpoor-Baltork, I.; Sadeghi, M. M.; Samani, Z. S. Indian J. Chem. 2001, 40B, 727. 